Christina J. Azevedo, $\mathrm{MD}, \mathrm{MPH}$

Eve Overton, BS Sankalpa Khadka, BS Jessica Buckley, BS Shuang Liu, PhD Mehul Sampat, PhD Orhun Kantarci, MD

Christine Lebrun Frenay, MD

Aksel Siva, MD

Darin T. Okuda, MD

Daniel Pelletier, MD

Correspondence to Dr. Azevedo: christina.azevedo@yale.edu

\title{
Early CNS neurodegeneration in radiologically isolated syndrome
} OPEN

\section{ABSTRACT}

Objective: Increasing evidence indicates that the thalamus may be a location of early neurodegeneration in multiple sclerosis (MS). Our objective was to identify the presence of gray matter volume loss and thinning in patients with radiologically isolated syndrome (RIS).

Methods: Sixty-three participants were included in this case-control study. Twenty-one patients with RIS were age- and sex-matched to 42 healthy controls in a 1:2 ratio. All participants underwent brain MRIs on a single 3T scanner. After lesion segmentation and inpainting, $1 \mathrm{~mm}^{3}$-isometric T1-weighted images were submitted to FreeSurfer (v5.2). Normalized cortical and deep gray matter volumes were compared between patients with RIS and controls using $t$ tests, and thalamic volumes were correlated with white matter lesion volumes using Pearson correlation. Exploratory cortical thickness maps were created.

Results: Although traditional normalized total gray and white matter volumes were not statistically different between patients with RIS and controls, normalized left $(0.0046 \pm 0.0005$ vs 0.0049 $\pm 0.0004, p=0.006)$, right $(0.0045 \pm 0.0005$ vs $0.0048 \pm 0.0004, p=0.008)$, and mean $(0.0045 \pm 0.0005$ vs $0.0049 \pm 0.0004, p=0.004)$ thalamic volumes were significantly lower in patients with RIS ( $n=21$, mean age $41.9 \pm 12.7$ years) than in controls ( $n=42$, mean age $41.4 \pm 11.2$ years). Thalamic volumes correlated modestly with white matter lesion volumes (range: $r=-0.35$ to -0.47 )

Conclusion: Our data provide novel evidence of thalamic atrophy in RIS and are consistent with previous reports in early MS stages. Thalamic volume loss is present early in CNS demyelinating disease and should be further investigated as a metric associated with neurodegeneration. Neurol Neuroimmunol Neuroinflamm 2015;2:e102; doi: 10.1212/NXI.0000000000000102

\section{GLOSSARY}

3D-IRSPGR = inversion recovery spoiled gradient echo T1-weighted isotropic volumetric sequence; CIS = clinically isolated syndrome; $\mathbf{M S}$ = multiple sclerosis; $\mathbf{R I S}=$ radiologically isolated syndrome; RRMS = relapsing-remitting MS; UCSF = University of California, San Francisco.

The thalamus is a major relay center with extensive cortical and subcortical anatomic connections. Increasing evidence suggests that the thalamus may be a location of early neurodegeneration in multiple sclerosis (MS). Thalamic atrophy has been reported in early relapsing-remitting MS (RRMS), ${ }^{1}$ clinically isolated syndrome (CIS) at presentation, ${ }^{2}$ and pediatric MS. ${ }^{3}$ Thalamic volume loss is likely due in part to disconnection caused by white matter lesions; using diffusion tensor imaging tractography, a relationship between thalamic volume and white matter lesion volume within thalamocortical projections has been observed. ${ }^{4}$ In addition to the thalamus, cortical gray matter may exhibit early volume loss or thinning. Regional cortical thinning has been identified as early as CIS. ${ }^{5}$

\footnotetext{
From the Department of Neurology (C.J.A., E.O., S.K., J.B., S.L., M.S., D.P.) and Department of Diagnostic Radiology (D.P.), Yale University,
New Haven, CT; Department of Neurology (O.K.), Mayo Clinic College of Medicine, Rochester, MN; Service de Neurologie (C.L.F.), Hospital

From the Department of Neurology (C.J.A., E.O., S.K., J.B., S.L., M.S., D.P.) and Department of Diagnostic Radiology (D.P.), Yale University,
New Haven, CT; Department of Neurology (O.K.), Mayo Clinic College of Medicine, Rochester, MN; Service de Neurologie (C.L.F.), Hospital Pasteur, Nice, France; Department of Neurology (A.S.), Cerrahpasa School of Medicine, University of Istanbul, Turkey; and Department of Neurology (D.T.O.), University of Texas Southwestern Medical Center, Dallas, TX.

Funding information and disclosures are provided at the end of the article. Go to Neurology.org/nn for full disclosure forms. The Article Processing Charge was paid by the authors.

This is an open access article distributed under the terms of the Creative Commons Attribution-Noncommercial No Derivative 3.0 License, which

permits downloading and sharing the work provided it is properly cited. The work cannot be changed in any way or used commercially.
} 
Radiologically isolated syndrome (RIS) is defined as incidentally identified white matter anomalies within the CNS that are suggestive of MS in healthy individuals who lack clinical symptomatology associated with CNS demyelination, after exclusion of other possible etiologies. ${ }^{6}$ Approximately 30\% of patients with RIS will have a sentinel clinical demyelinating event within 5 years of the identification of their abnormal $\mathrm{MRI}^{6,7}$; thus, for many patients, RIS represents the earliest visible manifestation of demyelinating disease, or a preclinical stage of MS.

The objective of this work was to investigate the presence of gray matter volume loss and thinning in patients with RIS using the FreeSurfer image analysis suite, an unbiased segmentation method. In addition to very robust and fully automated subcortical anatomic segmentation, FreeSurfer is capable of detecting submillimeter differences in cortical thickness between groups. ${ }^{8}$

METHODS Participants. Healthy individuals not exposed to a disease-modifying therapy fulfilling 2009 RIS criteria $^{6}$ were retrospectively identified at the University of California, San Francisco (UCSF) MS Center. For each participant with RIS, the first 2 available age- and sex-matched healthy controls were selected from a larger dataset (i.e., 1:2 ratio).

Standard protocol approvals, registrations, and patient consents. The protocol was approved by the Committee on Human Research at UCSF, and informed consent was obtained from all participants.

MRI acquisition. All study subjects were scanned on a single 3T GE Signa scanner using a standardized protocol. The protocol included an inversion recovery spoiled gradient echo $\mathrm{T} 1$-weighted isotropic volumetric sequence (3D-IRSPGR, $1 \times 1 \times 1 \mathrm{~mm}^{3}$, 180 slices, echo time/repetition time/inversion time $=2 / 7 / 400$ $\mathrm{ms}$, flip angle $=15^{\circ}, 256 \times 256 \times 180$ matrix, $240 \times 240 \times$ $180 \mathrm{~mm}^{3}$ field of view, number of excitations $=1$ ).

MRI postprocessing. The 3D-IRSPGR served as the input image into the postprocessing pipeline. T1 lesion masks were derived from manual segmentation of T1-visible white matter lesions, as described previously. ${ }^{6}$ None of the patients with RIS had visible thalamic lesions. MS lesions were inpainted ${ }^{9}$ on the 3D-IRSPGR prior to submitting the image to FreeSurfer. Inpainting was critical in order to avoid voxel misclassification due to juxtacortical MS lesions, which are of similar intensity to cortical gray matter on gradient echo images and can result in parcellation errors in FreeSurfer's output. The inpainted 3DIRSPGRs were submitted to FreeSurfer (v5.2; Desikan-Killiany atlas for surface parcellation and probabilistic atlas for subcortical segmentation). FreeSurfer output was reviewed by an experienced MRI reader/postprocessor (C.J.A.) and manually corrected using methods within FreeSurfer such as control points, white matter edits, and dura edits. Cases were rerun as needed until no further improvement was achieved by additional editing. On average, 3 runs per case were performed, but some cases required up to 7 runs. Particular attention was paid to error-prone regions such as the mesial temporal lobes.

Statistical analyses. Volumes (i.e., total gray matter, total white matter, deep gray nuclei) were extracted directly from FreeSurfer's automated segmentation and normalized for head size using the total intracranial volume (also taken from FreeSurfer). These values (unitless) were compared between patients with RIS and controls using $t$ tests. Among patients with RIS, thalamic volumes were correlated with log-transformed white matter lesion volumes using Pearson correlation. Statistical analyses were performed using SAS (v9.2). All hypothesis tests in this portion of the analysis were performed at a nominal $\alpha=0.05$ significance level without adjustment for multiple comparisons. Adjustment for multiple comparisons is generally performed when several independent hypothesis tests are conducted on one population. In this case, volumes for a given participant are likely to be correlated with each other and are thus not independent. As such, a correction would be too strict. Scientific judgment can be used to indicate where caution is warranted in interpreting results with $p<0.05$.

An exploratory, group-level, vertex-wise analysis of cortical thickness was performed using QDEC, a tool within FreeSurfer. Briefly, as part of its cortical parcellation, FreeSurfer reconstructs each individual's cortical surface using a meshwork of $\sim 150,000$ triangles. Each point of intersection between triangles is known as a vertex, and each vertex has an associated XYZ coordinate. QDEC creates an average cortical thickness map per group of participants and then generates a significant difference map by performing a generalized linear model at each vertex. Although we matched on age and sex, the final QDEC result was adjusted for both of these variables to avoid the possibility of residual confounding. QDEC's output was corrected for multiple comparisons using a Monte Carlo simulation procedure.

RESULTS Participant demographics. Baseline demographics for all participants are given in table 1 .

Comparison of volumes between patients with RIS and controls. Normalized total parenchymal (0.782 \pm 0.046 vs $0.798 \pm 0.035, p=0.12)$ and total white matter $(0.33 \pm 0.02$ vs $0.33 \pm 0.02, p=0.6)$ volumes were not statistically different between patients with RIS and controls. However, a trend toward lower normalized total gray matter volumes was seen in patients with RIS compared with controls ( $p=0.06$, table 2 ). When total gray matter was divided into cortical and subcortical gray matter, normalized cortical gray matter volume did not differ significantly between patients with RIS and controls $(p=0.13)$, but normalized subcortical gray matter volume was significantly lower in patients with RIS than in controls $(p=0.04)$. Among all subcortical gray matter structures, the thalamus was the main driver of this difference. Normalized left $(p=0.006)$, right $(p=0.008)$, and mean $(p=0.004)$ thalamic volumes were significantly lower in patients with RIS than in controls.

Correlation between thalamic volumes and white matter lesion volumes. Among patients with RIS, mean thalamic $(r=-0.42, p=0.06)$, left thalamic 


\begin{tabular}{|c|c|c|c|}
\hline \multicolumn{4}{|c|}{ Participant demographics } \\
\hline Demographic & RIS patients $(n=21)^{a}$ & Controls $(n=42)^{a}$ & p Value ${ }^{b}$ \\
\hline Age, y & $41.9 \pm 12.7$ & $41.4 \pm 11.2$ & 0.88 \\
\hline Age at RIS diagnosis, y & $39.9 \pm 12.8$ & NA & - \\
\hline Sex, F:M & $1.6: 1$ & $2.2: 1$ & 0.57 \\
\hline \multirow[t]{13}{*}{ Reason for initial MRI scan (n) } & Headache (6) & NA & - \\
\hline & Research control (2) & & \\
\hline & Stroke/TIA (2) & & \\
\hline & Trauma (2) & & \\
\hline & Allergies (1) & & \\
\hline & Aneurysm screen ${ }^{c}(1)$ & & \\
\hline & Anxiety (1) & & \\
\hline & Congenital cervical rib ${ }^{d}(1)$ & & \\
\hline & Dysmenorrhea (1) & & \\
\hline & Family history of MS (1) & & \\
\hline & Syncope (1) & & \\
\hline & Tinnitus (1) & & \\
\hline & Unknown (1) & & \\
\hline
\end{tabular}

Abbreviations: MS = multiple sclerosis; NA = not applicable; RIS = radiologically isolated syndrome.

a Mean \pm SD for continuous variables.

${ }^{b} p$ Value for $t$ test for continuous variables and $\chi^{2}$ test for categorical variables.

${ }^{c}$ For polycystic kidney disease.

${ }^{\mathrm{d} C e r v i c a l}$ spinal cord MRI showed MS-like anomalies and prompted brain MRI.

$(r=-0.35, p=0.13)$, and right thalamic $(r=-0.47$, $p=0.03$ ) volumes were modestly correlated with white matter lesion volumes. The correlations were in the expected direction and generally exhibited a trend toward statistical significance. The asymmetry in the strength of the correlation for right and left thalami was not expected a priori and may have been due to chance.

Cortical thinning. The final result from QDEC analysis is shown in the figure. An area measuring approximately $3 \mathrm{~cm}$ in the right superior and inferior parietal gyri (Desikan-Killiany labels) was significantly thinner in patients with RIS than in controls. It was present at Monte Carlo thresholds of $p<0.05$ and $p<0.01$, and was stable up to a threshold of $p<0.005$.

DISCUSSION These data provide novel in vivo evidence to suggest that tissue loss may precede clinical manifestations of CNS demyelination. These findings add to previous work reporting that thalamic atrophy is present in early stages of CNS demyelinating disease, including early RRMS, ${ }^{1} \mathrm{CIS},{ }^{2}$ and pediatric MS. ${ }^{3}$

Thalamic atrophy is likely due to several converging aspects of MS disease pathology. Although we did not see thalamic lesions in our RIS population, thalamic demyelinating lesions $s^{10}$ and iron deposition ${ }^{11}$ likely contribute to thalamic volume loss. In addition, neuronal density has been shown to be reduced in nonlesional thalamic tissue, ${ }^{12}$ which may be driven by disconnection from white matter lesions in the various tracts that project from and into the thalamus ${ }^{4}$ (e.g., thalamocortical, opticothalamic, and spinothalamic projections). Further studies at very high field strengths examining the interplay of these factors, including the contribution of thalamic demyelinating lesions and iron deposition to thalamic volume loss, will be of interest.

Although others have reported decreased whole brain and cortical gray matter volumes in patients with RIS compared with healthy controls, ${ }^{13}$ we did not see such differences in our data. Our findings are consistent with a CIS cohort ${ }^{2}$ in which there was no difference in normalized whole brain or gray matter volumes but there was a reduction in thalamic volume using voxel-based morphometry. To our knowledge, there are no other published studies examining cortical thickness in patients with RIS. We report our finding of a $\sim 3 \mathrm{~cm}$ area of thinning in the right superior and inferior parietal gyri but regard it as a preliminary finding without a specific rationale for

Table 2 Normalized gray matter regions in patients with radiologically isolated syndrome (RIS) and controls

$\begin{array}{llll}\text { Regions normalized for total intracranial volume }^{a} & \text { RIS }(n=21)^{b} & \text { Controls }(n=42)^{b} & p \text { Value }^{\mathbf{b}} \\ \text { Total gray matter }^{d} & 0.451 \pm 0.032 & 0.464 \pm 0.023 & 0.06 \\ \text { Cortical gray matter } & 0.325 \pm 0.024 & 0.333 \pm 0.019 & 0.13 \\ \text { Subcortical gray matter } & 0.126 \pm 0.010 & 0.131 \pm 0.008 & 0.04^{\mathrm{e}} \\ \quad \text { Mean thalamic } & 0.0045 \pm 0.0005 & 0.0049 \pm 0.0004 & 0.004^{\mathrm{e}} \\ \text { Left thalamic } & 0.0046 \pm 0.0005 & 0.0049 \pm 0.0004 & 0.006^{\mathrm{e}} \\ \text { Right thalamic } & 0.0045 \pm 0.0005 & 0.0048 \pm 0.0004 & 0.008^{\mathrm{e}}\end{array}$

${ }^{\text {a }}$ Results are unitless (normalized volume $=$ volume $\mathrm{mm}^{3} /$ total intracranial volume $\mathrm{mm}^{3}$ ) and expressed as proportion.

${ }^{b}$ Mean \pm SD.

${ }^{c} p$ Value for $t$ test.

${ }^{\mathrm{d}}$ Total gray matter excludes brainstem and cerebellum.

e Statistically significant at alpha $=0.05$. 
Figure

Vertex-wise significant difference map (QDEC) of cortical thickness between patients with RIS and controls

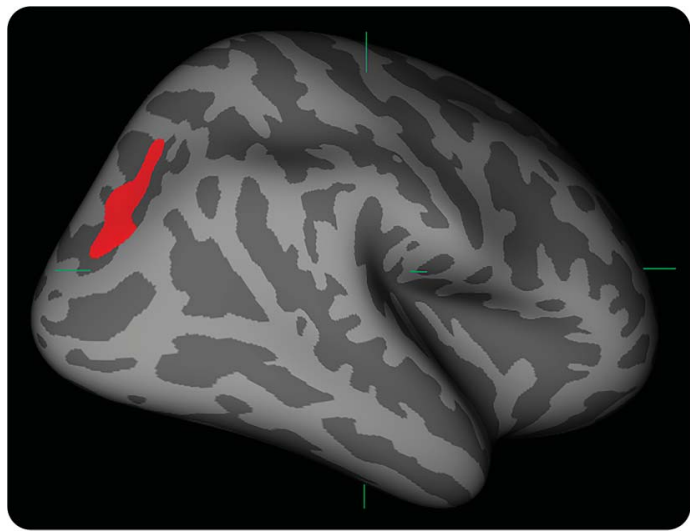

An area measuring approximately $3 \mathrm{~cm}$ in the right superior and inferior parietal gyri (Desikan-Killiany labels, in red) was significantly thinner in patients with radiologically isolated syndrome (RIS) than in controls at a Monte Carlo threshold of $p<0.005$ and adjusted for age and sex.

why these particular gyri are affected in our dataset. In a group of patients with CIS, this region was not significantly different from controls using different FreeSurfer methods and input images. ${ }^{5}$ Further studies examining whole brain and regional gray matter volumes and cortical thickness in early demyelinating disease will be of interest in order to replicate these findings.

Our study has certain limitations, including a small sample size and a single-center design. In addition, cortical lesions are of great interest in the field currently but are generally not visible on conventional MRIs, including the IRSPGRs we used; as such, this work is not able to address the impact of cortical lesions on cortical thinning. Despite these limitations, high-resolution images and a careful MRI postprocessing approach were used, and the results are felt to be robust.

The reflection of multiple aspects of MS pathology, the presence of thalamic volume loss in early disease, the relative ease of measuring thalamic volume by various methods, and the observed correlation of thalamic volume with cognition ${ }^{14}$ make thalamic volume an attractive potential MRI clinical trial metric associated with the neurodegenerative aspect of MS and potentially RIS. Further studies are needed to investigate the temporal evolution and the effect of disease-modifying therapies on thalamic volume loss. In the context of RIS, larger longitudinal studies are currently under way. Although there may be other areas of gray matter volume loss or thinning in RIS, thalamic volume will be of particular interest in these studies and will be examined as a potential predictor of conversion to CIS/MS.

\section{AUTHOR CONTRIBUTIONS}

Dr. Azevedo contributed to the study concept and design, MRI postprocessing, statistical analysis and interpretation of data, and preparation/ revision of the manuscript. Ms. Overton, Mr. Khadka, Ms. Buckley, Dr. Liu, and Dr. Sampat contributed to MRI postprocessing and revision of the manuscript for intellectual content. Dr. Kantarci, Dr. Lebrun Frenay, and Dr. Siva contributed to critical revision of the manuscript for intellectual content. Dr. Okuda contributed to data collection and patient enrollment, acquisition of data, and critical revision of the manuscript for intellectual content. Dr. Pelletier contributed to study concept and design, acquisition of data, MRI postprocessing, data analysis and interpretation, critical revision of the manuscript for intellectual content, and overall study supervision.

\section{ACKNOWLEDGMENT}

The authors thank the research subjects who generously agreed to serve as study participants and the research coordinators who assisted in data collection.

\section{STUDY FUNDING}

This research was supported by the NIH (National Institute of Neurological Disorders and Stroke R01NS062885 to D.P.), the National Multiple Sclerosis Society (Sylvia Lawry Physician Fellowship Award FP1778-A-1 to C.J.A.), and the Race to Erase MS Foundation (14-003399 to C.J.A.).

\section{DISCLOSURE}

C.J. Azevedo served on a medical advisory board for Genzyme and received research support from the Nancy Davis Foundation. E. Overton received research support from the Foundation of the Consortium of Multiple Sclerosis Centers. S. Khadka, J. Buckley, S. Liu report no disclosures. M. Sampat is employed by Bioclinical/Synarc. O. Kantarci received speaker honoraria from Novartis; performed a grant review for the National Multiple Sclerosis Society; received research support from the European Regional Development Fund, NIH, Mayo Foundation, Hilton Foundation, Minnesota Partnership for Biotechnology and Medical Genomics, and National Multiple Sclerosis Society; has given a scientific presentation at a meeting supported by Teva Pharmaceuticals; and is on the data safety monitoring board for Takeda Global Research and Development Center, Pfizer Inc, and Janssen Alzheimer Immunotherapy. C.L. Frenay served on the scientific advisory board for Biogen Idec, Merck Serono, Genzyme, Almirall, Allergan, Teva, Sanofi, and Bayer Schering; received travel funding and/or speaker honoraria from Biogen Idec, Merck Serono, Genzyme, Almirall, Allergan, Teva, Sanofi, and Bayer Schering; and is an editorial advisory board member for Revue Neurologique. A. Siva received travel funding and/or registration coverage from Merck Serono, Biogen Idec/Gen Pharma of Turkey, Novartis, and Teva; consulted for Biogen Idec, Novartis, and Teva; presented for Excemed; and received research support from The Scientific and Technological Research Council of Turkey. D. T. Okuda received travel funding and/or speaker honoraria from Acorda Therapeutics, Genzyme, Multiple Sclerosis Association of America, MS Cure Fund, and Teva Neuroscience; has consulted for Genzyme and Teva Neuroscience; is on the speakers' bureau for Acorda Therapeutics, Genzyme, and Teva Neuroscience; and received research support from Biogen Idec. D. Pelletier has consulted for CNS Imaging Consultant, LLC and received research support from Biogen Idec, Hoffmann-LaRoche, and NIH/National Institute of Neurological Disorders and Stroke. Go to Neurology.org/nn for full disclosure forms.

Received November 13, 2014. Accepted in final form March 5, 2015.

\section{REFERENCES}

1. Wylezinska M, Cifelli A, Jezzard P, Palace J, Alecci M, Matthews PM. Thalamic neurodegeneration in relapsingremitting multiple sclerosis. Neurology 2003;60:19491954.

2. Henry RG, Shieh M, Okuda DT, Evangelista A, GornoTempini ML, Pelletier D. Regional grey matter atrophy in 
clinically isolated syndromes at presentation. J Neurol Neurosurg Psychiatry 2008;79:1236-1244.

3. Aubert-Broche B, Fonov V, Ghassemi R, et al. Regional brain atrophy in children with multiple sclerosis. Neuroimage 2011;58:409-415.

4. Henry RG, Shieh M, Amirbekian B, Chung W, Okuda DT, Pelletier D. Connecting white matter injury and thalamic atrophy in clinically isolated syndromes. J Neurol Sci 2009;282:61-66.

5. Calabrese M, Atzori M, Bernardi V, et al. Cortical atrophy is relevant in multiple sclerosis at clinical onset. J Neurol 2007;254:1212-1220.

6. Okuda DT, Mowry EM, Beheshtian A, et al. Incidental MRI anomalies suggestive of multiple sclerosis: the radiologically isolated syndrome. Neurology 2009;72:800-805.

7. Okuda DT, Siva A, Kantarci O, et al. Radiologically isolated syndrome: 5-year risk for an initial clinical event. PLoS One 2014;9:e90509. doi: 10.137/journal.pone. 0090509.

8. Dale AM, Fischl B, Sereno MI. Cortical surface-based analysis. I: Segmentation and surface reconstruction. Neuroimage 1999;9:179-194.
9. Sdika M, Pelletier D. Nonrigid registration of multiple sclerosis brain images using lesion inpainting for morphometry or lesion mapping. Hum Brain Mapp 2009; 30:1060-1067.

10. Vercellino M, Plano F, Votta B, Mutani R, Giordana MT, Cavalla P. Grey matter pathology in multiple sclerosis. J Neuropathol Exp Neurol 2005;64:1101-1107.

11. Zivadinov $\mathrm{R}$, Heininen-Brown $\mathrm{M}$, Schirda $\mathrm{CV}$, et al. Abnormal subcortical deep-gray matter susceptibilityweighted imaging filtered phase measurements in patients with multiple sclerosis: a case-control study. Neuroimage 2012;59:331-339.

12. Cifelli A, Arridge M, Jezzard P, Esiri MM, Palace J, Matthews PM. Thalamic neurodegeneration in multiple sclerosis. Ann Neurol 2002;52:650-653.

13. De Stefano N, Stromillo ML, Rossi F, et al. Improving the characterization of radiologically isolated syndrome suggestive of multiple sclerosis. PLoS One 2011;6:e19452. doi: 10.1371/journal.pone.0019452.

14. Houtchens MK, Benedict RH, Killiany R, et al. Thalamic atrophy and cognition in multiple sclerosis. Neurology 2007;69:1213-1223. 


\section{Neurology \\ Neuroimmunology \& Neuroinflammation}

Early CNS neurodegeneration in radiologically isolated syndrome

Christina J. Azevedo, Eve Overton, Sankalpa Khadka, et al.

Neurol Neuroimmunol Neuroinflamm 2015;2;

DOI 10.1212/NXI.0000000000000102

This information is current as of April 9, 2015

\section{Updated Information \& Services}

References

Citations

Subspecialty Collections

Permissions \& Licensing

Reprints including high resolution figures, can be found at:

http://nn.neurology.org/content/2/3/e102.full.html

This article cites 14 articles, 1 of which you can access for free at: http://nn.neurology.org/content/2/3/e102.full.html\#\#ref-list-1

This article has been cited by 9 HighWire-hosted articles: http://nn.neurology.org/content/2/3/e102.full.html\#\#otherarticles

This article, along with others on similar topics, appears in the following collection(s):

All Demyelinating disease (CNS)

http://nn.neurology.org//cgi/collection/all_demyelinating_disease_cns MRI

http://nn.neurology.org//cgi/collection/mri

Multiple sclerosis

http://nn.neurology.org//cgi/collection/multiple_sclerosis

Information about reproducing this article in parts (figures,tables) or in its entirety can be found online at:

http://nn.neurology.org/misc/about.xhtml\#permissions

Information about ordering reprints can be found online: http://nn.neurology.org/misc/addir.xhtml\#reprintsus

Neurol Neuroimmunol Neuroinflamm is an official journal of the American Academy of Neurology.

Published since April 2014, it is an open-access, online-only, continuous publication journal. Copyright $\odot$ 2015 American Academy of Neurology. All rights reserved. Online ISSN: 2332-7812.

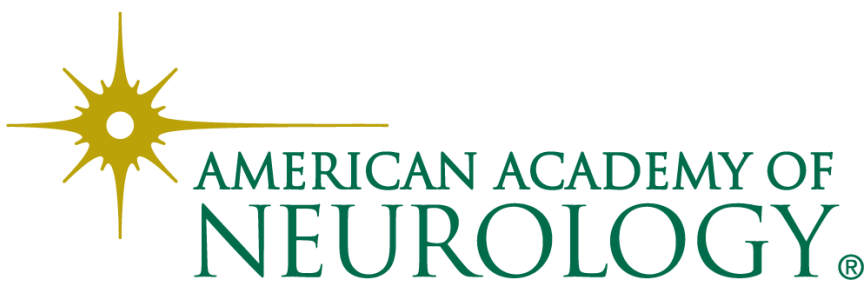

